\title{
Numerical Simulation Analysis and Experimental Research on Damping Performance of a Novel Magnetic Fluid Damper
}

\author{
Wenrong Yang $\mathbb{D},,^{1,2}$ Dejie Wei $\mathbb{D},{ }^{1}$ Jianzheng Su $\mathbb{D}^{1},,^{1}$ Xiaorui Yang $\mathbb{D},{ }^{3}$ and Qingxin Yang $\mathbb{B D}^{1}$ \\ ${ }^{1}$ State Key Laboratory of Reliability and Intelligence of Electrical Equipment, Hebei University of Technology, \\ Tianjin 300130, China \\ ${ }^{2}$ Key Laboratory of Electromagnetic Field and Electrical Apparatus Reliability of Hebei Province, Hebei University of Technology, \\ Tianjin 300130, China \\ ${ }^{3}$ School of Electrical Engineering, Hebei University of Science and Technology, Shijiazhuang 050018, China
}

Correspondence should be addressed to Wenrong Yang; wryang@hebut.edu.cn

Received 11 December 2020; Revised 18 January 2021; Accepted 28 January 2021; Published 8 February 2021

Academic Editor: Luigi Di Sarno

Copyright (c) 2021 Wenrong Yang et al. This is an open access article distributed under the Creative Commons Attribution License, which permits unrestricted use, distribution, and reproduction in any medium, provided the original work is properly cited.

\begin{abstract}
Considering the low-frequency and large-amplitude vibration characteristics of the high-rise structure, a tuned magnetic fluid rolling-ball damper is proposed to suppress the vibration of the structure. By adjusting the external magnetic field to control the natural rolling frequency of the ball, the purpose of tuning vibration reduction is achieved. Firstly, the working principle of the damper is theoretically analysed, a three-dimensional (3D) magnetic-fluid-solid multiphysical field coupling mathematical model of the damper is established and the governing equations of multiphysical field coupling are derived. Secondly, the magnetic field distribution and operating characteristics of the damper are simulated and analysed. Finally, the effectiveness of the model is verified by experiments, and the damping performance of the damper with two kinds of magnetic fluid is tested and compared. The results show that the magnetic-fluid-solid multiphysical field coupling model can accurately simulate the working characteristics of the damper. The maximum damping force of the damper is about $12 \%$ of the elastic force of the structure, which can increase the damping ratio of the structure by about two times, effectively reduce the vibration response time, and suppress the vibration of the high-rise structure.
\end{abstract}

\section{Introduction}

Ferrofluid, also called magnetic fluid, is a kind of colloidal stable liquid composed of magnetic nanoparticles, carrier fluid, and surfactant. The surfactant is coated on the surface of magnetic nanoparticles to prevent the mutual attraction of solid nanoparticles from causing aggregation, and due to Brownian motion, magnetic nanoparticles are uniformly dispersed in carrier liquid $[1,2]$. Magnetic fluid has unique magnetic and hydrodynamic properties $[3,4]$. Under the effect of magnetic field, magnetic fluid is magnetized and the magnetic torque of magnetic nanoparticles increases to resist the viscous torque, which leads to the increase of friction force between the magnetic nanoparticles and the carrier liquid $[5,6]$, showing the increase of the apparent viscosity of the magnetic fluid, which is called magnetoviscous characteristic $[7,8]$. At the same time, magnetic fluid also exhibits superparamagnetism. When the external magnetic field is removed, there is no remanence and coercivity, so it has outstanding controllability and response characteristics in damping applications [9]. Magnetic fluids should not be confused with magnetorheological fluid (MRF); MRF had been widely used in damping in early studies due to the large damping force $[10,11]$. However, MRF is prone to aggregation and precipitation, and it has the disadvantage of poor stability [12]. Compared to MRF, magnetic fluid has higher stability.

As a special smart nanomaterial, magnetic fluid was used in damping in the early research period due to its special magnetoviscous characteristic and colloidal 
stability. In 1967, NASA was the first one to propose a magnetic fluid viscous damper, which effectively suppressed the low-frequency vibration in RAE satellite [13]. In 1978, Moskowitz et al. [14] put forward a magnetic fluid inertia damper to reduce the vibration of stepping motor shaft and optimized the structure, so that the magnetic fluid was attracted to the maximum magnetic field area without the need of mechanical fluid seal. In 1987, Katsuto et al. [15] proposed a piston-type magnetic fluid damper, which was combined with spring to control the vibration of the isolating table. Later, Yang et al. [16] wound electromagnetic induction coils around the piston-type magnetic fluid damper to achieve the purpose of active vibration control and carried out fluid-solid-thermal multiphysical field coupling analysis on the damper. Sawada et al. [17-21] developed the analysis model and numerical simulation of the semiactive tuned magnetic fluid damper and carried out a series of experiments to verify the effectiveness of the damper. Such semiactive dampers are expected. Li et al. [22, 23] proposed a magnetic fluid dynamic shock absorber based on second-order buoyancy, carried out dynamic modeling and a large number of experimental studies, and optimized the structure of the damper. At present, some magnetic fluid dampers have been used in mechanics, instrumentation, and aerospace fields, and some of them are still in the stage of theoretical analysis [24,25]. Due to its unique magnetoviscous characteristic and stability, magnetic fluid damper is still one of its most potential applications.

In this paper, a tuned magnetic fluid rolling-ball damper is proposed for tower structures with high flexibility and low vibration frequency $[26,27]$. Through the establishment of the magnetic-fluid-solid multiphysical field coupling model of the damper and the simulation study, the working characteristics of rolling ball and magnetic fluid were explored, and the natural sloshing frequency of the damper was theoretically and numerically analysed. The vibration experiments were carried out to verify the effectiveness of the model and test the damping performance of the damper with two kinds of magnetic fluid. The damper has the advantages of simple structure, high stability, fast vibration response, and the same performance in each vibration direction. The damper also has a good application prospect in vibration control of high-rise structures.

\section{Multiphysical Field Coupling Mathematical}

2.1. Structure and Operation Principle. Figure 1 shows the $3 \mathrm{D}$ structure section of the tuned magnetic fluid rolling-ball damper, which consists of a nonmagnetic cylindrical vessel with a hemispherical groove, a nonmagnetic hollow ball, a pedestal, and an induction coil. The induction coil is wound outside of the nonmagnetic cylindrical vessel to provide a magnetic field for magnetic fluid. A certain amount of magnetic fluid is placed inside the nonmagnetic hollow ball, and the nonmagnetic hollow ball is sealed by a screw.

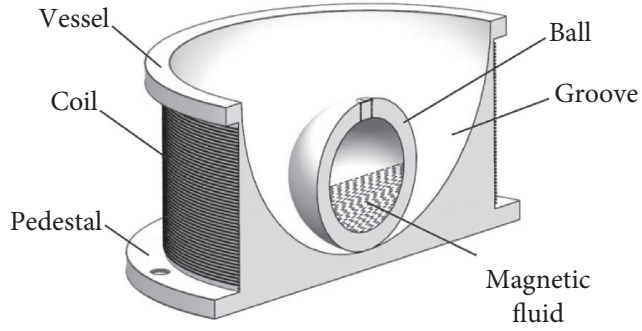

Figure 1: The 3D structure section of the tuned magnetic fluid rolling-ball damper.

The damper is connected with the high-rise structure through the pedestal. When the high-rise structure is excited, the damper will vibrate; together, after they are tuned, they will be in a state of reverse resonance. At this time, the damper will exert a reverse damping force on the structure, so as to suppress the vibration of the structure. Based on the magnetoviscosity characteristic of magnetic fluid, the viscosity of magnetic fluid can be changed by controlling the external magnetic field, and the frictional shear stress between the boundary layer of magnetic fluid and the inner surface of the ball can be changed, so as to control the natural rolling frequency of the ball and change the resonance characteristics of the structure to achieve the purpose of tuning vibration reduction. According to the Lagrange equation, the damping vibration reduction system is analysed dynamically and the ball's natural rolling frequency can be expressed as [28]

$$
f=\frac{1}{2 \pi} \sqrt{\frac{\left(m_{a}+m_{l}\right) g(R-r)-m_{p 1} \cdot 1 / 2 \mu_{0} n_{1} I R / \sqrt{R^{2}+r^{2}}}{\left(5 / 3 m_{a}+m_{l}\right)(R-r)^{2}}},
$$

where $m_{\mathrm{a}}$ and $m_{l}$ are the mass of the ball and magnetic fluid, respectively; $R$ is the radius of the groove; $r$ is the radius of the ball; $m_{p 1}$ is the magnetic moment of magnetic fluid; $\mu_{0}$ is the vacuum permeability; $n_{1}$ is the turn number of the induction coil; and $I$ is the input current.

According to equation (1), after the size of the damper is determined, the natural rolling frequency of the ball can be adjusted by only changing the input current in the induction coil to achieve the purpose of tuning vibration reduction.

2.2. Multiphysical Field Coupling Analysis. During the operation of the damper, the ball will drive the magnetic fluid to roll in the hemispherical groove, and the external magnetic field is controlled by changing the input current in the induction coil, so the damper will be in the magnetic-fluid-solid coupling field. The important feature of fluid-solid coupling is the interaction between two-phase media: the solid deforms or moves under the action of fluid dynamic load, which in turn affects the flow field and changes the distribution of fluid load. 
According to Newton's second law, for any undeformed motion structure with volume $V_{0}$, the integral form of momentum conservation can be expressed as

$$
\frac{\mathrm{d}}{\mathrm{d} t} \int_{V_{0}} \rho_{\text {solid }} \mathbf{v}_{\text {solid }} \mathrm{d} V=\int_{V_{0}} \mathbf{f}_{\mathrm{V}} \mathrm{d} V+\oint \mathbf{T} \cdot \mathrm{d} \mathbf{s} .
$$

According to the divergence law, $\oint \mathbf{T} \cdot \mathrm{d} \mathbf{s}=\int_{V_{0}} \nabla \cdot \mathbf{T} \mathrm{d} V$, the governing equation of rolling ball can be written as

$$
\rho_{\text {solid }} \frac{\partial^{2} \mathbf{u}}{\partial t^{2}}=\mathbf{f}_{\mathrm{V}}+\nabla \cdot \mathbf{T}
$$

where $\rho_{\text {solid }}$ is the density of the ball, $\mathbf{u}$ is the displacement vector of solid structure motion, $\mathbf{f}_{\mathrm{V}}$ is the volume force, and $\mathrm{T}$ is the traction force per unit area.

When the ball rolls in the groove, the tractive force mainly includes the friction force between outer surface of the ball and groove surface and the friction force between inner surface of the ball and the magnetic fluid boundary layer, which can be expressed by

$$
\mathbf{T}=-\xi\left(m_{a}+m_{l}\right) g \frac{R-r}{r} \mathbf{e}_{\boldsymbol{\omega}} \cos \theta-\eta_{\mathrm{H}} \frac{(R-r) \boldsymbol{\omega} \sqrt{\mathrm{Re}_{\mathrm{L}}}}{5 L r},
$$

where $\xi$ is the friction coefficient between the rolling ball and the groove, $\omega$ is the angular velocity of the ball, $\mathbf{e}_{\omega}$ is the angular velocity unit vector of the ball, $\theta$ is the angular displacement of the ball relative to the centre of the groove, $\eta_{H}$ is the dynamic viscosity of magnetic fluid, and $\operatorname{Re}_{L}$ is the Reynolds number of magnetic fluid flow.

As an incompressible fluid, magnetic fluid satisfies the continuity equation and the Navier-Stokes equation:

$$
\begin{gathered}
\nabla \cdot \mathbf{v}_{\text {fluid }}=0, \\
\rho_{\text {fluid }} \frac{\partial \mathbf{v}_{\text {fluid }}}{\partial t}+\rho\left(\mathbf{v}_{\text {fluid }} \cdot \nabla\right) \mathbf{v}_{\text {fluid }}=\mathbf{f}_{g}+\mathbf{f}_{p}+\mathbf{f}_{\eta},
\end{gathered}
$$

where $\rho_{\text {fluid }}$ is the density of magnetic fluid, $\mathbf{f}_{g}$ is the gravity per unit pressure volume of magnetic liquid, $\mathbf{f}_{p}$ is the gradient per unit pressure volume of magnetic fluid, $\mathbf{f}_{p}=-\nabla p, p$ is the pressure per unit pressure volume of magnetic fluid, and $\mathbf{f}_{\eta}$ is the viscous force of magnetic fluid, $\mathbf{f}_{\eta}=\eta_{\mathrm{H}} \nabla^{2} \mathbf{v}_{\text {fluid }}$, which can be changed by an external magnetic field.

The magnetic fluid is sealed inside the ball. The twophase field method is used to track the liquid level change of magnetic fluid [29-31], and the governing equations can be written as

$$
\begin{aligned}
\frac{\partial \phi}{\partial t}+\mathbf{v}_{\text {fluid }} \cdot \nabla \phi & =\nabla \cdot \frac{\gamma \lambda}{\varepsilon^{2}} \nabla \psi, \\
\psi & =-\nabla \cdot \varepsilon^{2} \nabla \phi+\left(\phi^{2}-1\right) \phi,
\end{aligned}
$$

where $\lambda$ is the mixing energy density and $\varepsilon$ is a capillary width that scales with the thickness of the interface. These two parameters are related to the surface tension coefficient $\sigma$; the expression is

$$
\sigma=\frac{2 \sqrt{2}}{3} \frac{\lambda}{\bar{\varepsilon}}
$$

The fluid-solid coupling governing equation is composed of equations (3) and (4), continuity equation (5), and Navier-Stokes equation (6). There is a fluid-solid coupling surface between the spherical shell and the magnetic fluid, which satisfies two boundary conditions: the normal velocity is continuous and the normal force is continuous.

$$
\begin{aligned}
& \mathbf{v}_{f} \cdot \mathbf{n}_{f}=\mathbf{v}_{s} \cdot \mathbf{n}_{f}=-\mathbf{v}_{s} \cdot \mathbf{n}_{s}, \\
& \mathbf{P}_{f} \cdot \mathbf{n}_{f}=\boldsymbol{\sigma}_{s} \cdot \mathbf{n}_{s},
\end{aligned}
$$

where $\mathbf{v}_{f}$ and $\mathbf{v}_{s}$ represent the velocity vectors of fluid and solid on the fluid-solid coupling surface, respectively. $\mathbf{n}_{f}$ and $\mathbf{n}_{s}$ represent the unit external normal vector of fluid boundary and the unit external normal vector of solid boundary on the fluid-solid coupling surface, respectively, and the direction is opposite. $\mathbf{P}_{f}$ and $\sigma_{s}$ represent stress tensors of fluid and solid, respectively.

The magnetic field in the damper is provided by the induction coil and ignoring the displacement current in the material. Through the geometric analysis of the coil, the current density of the DC coil is determined by the coil turns and the input current; the equation can be written as

$$
\mathbf{J}_{e}=\frac{N I_{\text {coil }}}{S} \mathbf{e}_{\text {coil }} \text {, }
$$

where $\mathbf{e}_{\text {coil }}$ is the unit vector perpendicular to the coil section and in the same direction as the current.

According to Ampere's circuital law and the vector magnetic potential, the governing equations of magnetic field generated by electromagnetic coil are expressed as

$$
\begin{aligned}
\nabla \times \mathbf{H} & =\mathbf{J}_{e} \\
\mathbf{B} & =\nabla \times \mathbf{A}, \\
\mathbf{H} & =\frac{\mathbf{B}}{\mu}-\mathbf{M} .
\end{aligned}
$$

The magnetic fluid is magnetized and its viscosity changes in the magnetic field. Magnetic field, fluid field, and solid field are coupled through dynamic viscosity of magnetic fluid. The magnetoviscous characteristic curve measured by rotary viscometer is shown in Figure 2. Under the action of $0 \mathrm{mT}-30 \mathrm{mT}$ magnetic field, the magnetic fluid viscosity changes from $5 \mathrm{mPa}^{*}$ s to $18 \mathrm{mPa}^{*}$. 


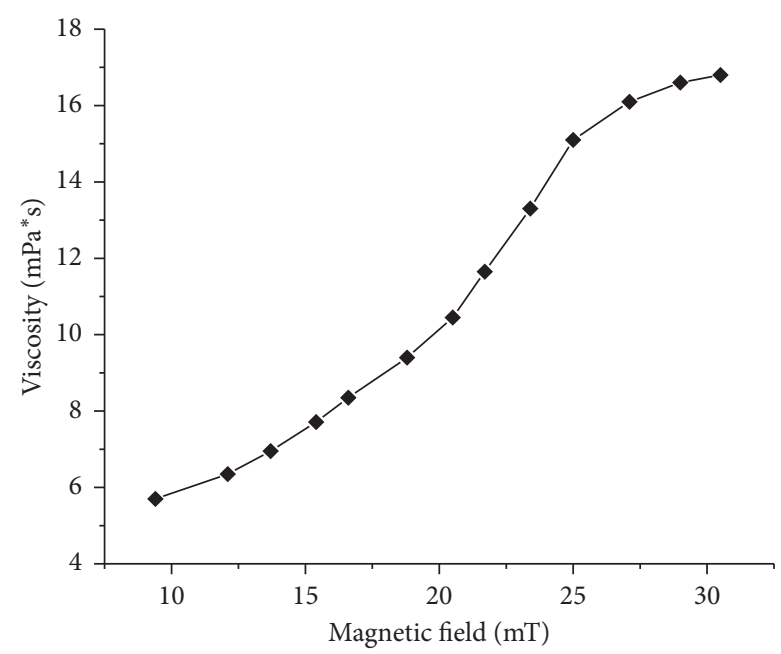

Figure 2: The magnetoviscous characteristic curve.

According to equations (3), (4), (6), and (12)-(15), the governing equations of the damper's magnetic-fluid-solid multiphysical field coupling model can be written as

$$
\left\{\begin{array}{l}
\rho_{\text {solid }} \frac{\partial^{2} \mathbf{u}_{\text {solid }}}{\partial t^{2}}=\mathbf{f}_{\mathbf{V}}+\nabla \cdot\left(-\xi\left(m_{a}+m_{l}\right) g \frac{R-r}{r} \mathbf{e}_{\boldsymbol{\omega}} \cos \theta-\eta_{H} \frac{(R-r) \boldsymbol{\omega} \sqrt{\mathrm{Re}_{L}}}{5 L r}\right) \\
\rho_{\text {fluid }} \frac{\partial \mathbf{v}_{\text {fluid }}}{\partial t}+\rho_{\text {fluid }}\left(\mathbf{v}_{\text {fluid }} \cdot \nabla\right) \mathbf{v}_{\text {fluid }}=\mathbf{f}_{g}-\nabla p+\eta_{\mathrm{H}} \nabla^{2} \mathbf{v}_{\text {fluid }}, \\
\nabla \times\left(\frac{1}{\mu}(\nabla \times \mathbf{A})-\mathrm{M}\right)=\frac{N I_{\text {coil }}}{S} \mathbf{e}_{\text {coil }} .
\end{array}\right.
$$

2.3. Multiphysical Field Coupling Simulation. Based on the governing equations of the damper's magnetic-fluid-solid multiphysical field coupling model, the 3D multiphysical field coupling simulation model is established by the finite element method, as shown in Figure 3. In the simulation model, the radius of groove and ball are $100 \mathrm{~mm}$ and $40 \mathrm{~mm}$, respectively. The mass and density of kerosene-based $\mathrm{Fe}_{3} \mathrm{O}_{4}$ magnetic fluid are $175.5 \mathrm{~g}$ and $1.41 \mathrm{~g} / \mathrm{cm}^{3}$. The DC coil is set by using the multiturn coil node; the turn number is 400 , and the diameter of coil wire is $1 \mathrm{~mm}$. The shell and rolling ball of the damper are made of resin.

Based on the magnetoviscous characteristic curve of magnetic fluid, as shown in Figure 2, the magnetic field inside the damper should be numerically calculated and analysed firstly. Figure 4(a) shows the magnetic field distribution inside groove of the damper with $2 \mathrm{~A}$ input current and Figure 4(b) shows the magnetic flux density on the central axis of the damper with the input current ranges from $0 \mathrm{~A}$ to $4 \mathrm{~A}$. The magnetic field from the bottom to the top increases first and then decreases along the central axis of the groove and has a good symmetry along the central axis, which meets the magnetic field requirement for viscosity changes. Therefore, no matter where the ball rolls in the groove, the magnetic field can change the viscosity and control the rolling frequency.

An initial horizontal excitation is applied to the damper, and the ball will roll along the horizontal exciting direction in the groove. When the input current of the induction coil is $3.1 \mathrm{~A}$, the relationship curve between displacement of the ball and time is shown in Figure 5. It is obvious that the natural vibration frequency of the rolling ball is $1.61 \mathrm{~Hz}$, which is consistent with the theoretical calculation according to equation (1). Figure 6 shows the magnetic fluid level change in a vibration period, and its sloshing frequency is close to the rolling frequency of the ball, which indicates that the magnetic fluid is in the turbulent state during operation. In this case, the friction energy consumption between the magnetic nanoparticles and the carrier liquid is faster, and the vibration reduction effect is better.

On the basis of the simulation of the damper, the damper is fixed on a single-degree-of-freedom high-rise structure for vibration reduction simulation. The high-rise frame structure is made of $1 \mathrm{~mm}$-thick phosphor copper plate, and its stiffness $k$ is $7693 \mathrm{~N} / \mathrm{m}$. According to the simulation results, the height is $460 \mathrm{~mm}$ and the natural frequency is $1.61 \mathrm{~Hz}$. An initial excitation is applied to the top of the structure; the 


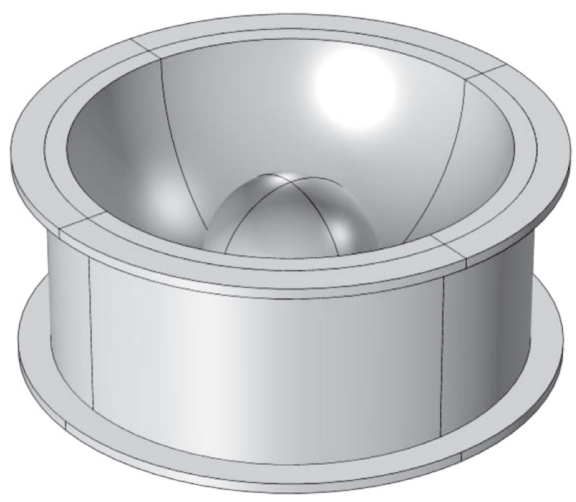

Figure 3: Simulation model of the damper.

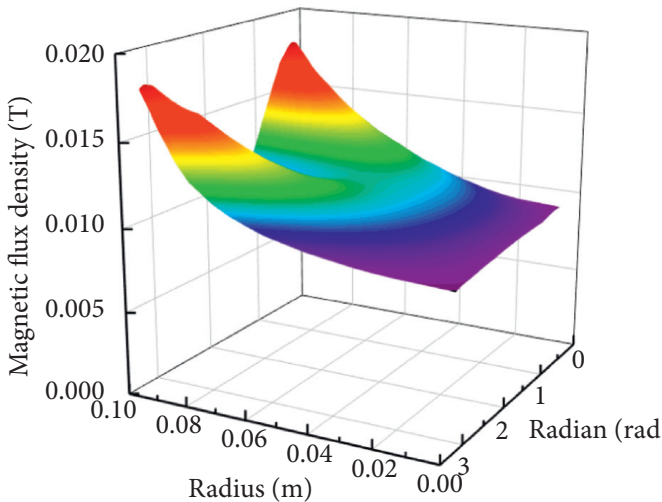

(a)

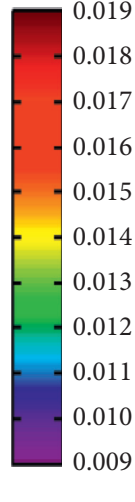

0.009

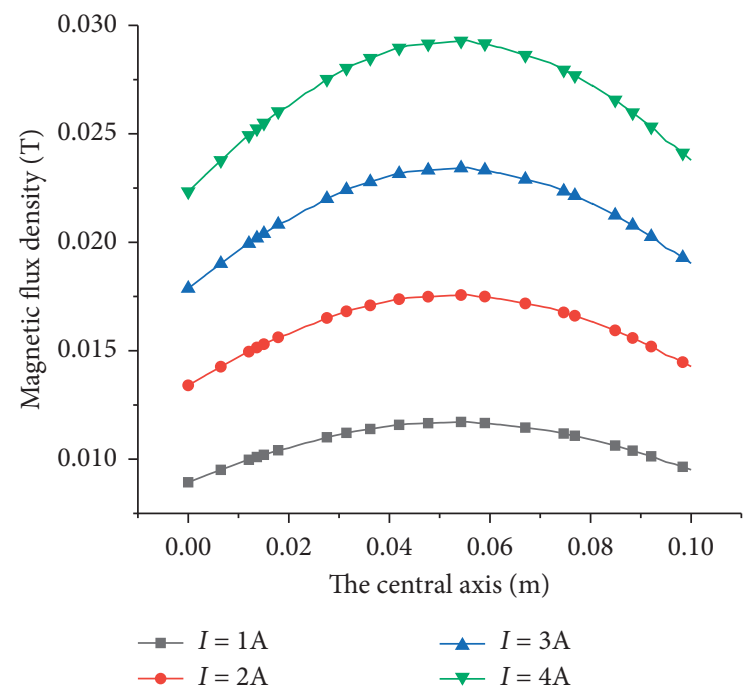

(b)

Figure 4: The magnetic field distribution. (a) Inside the groove of the damper. (b) On the central axis of the damper.

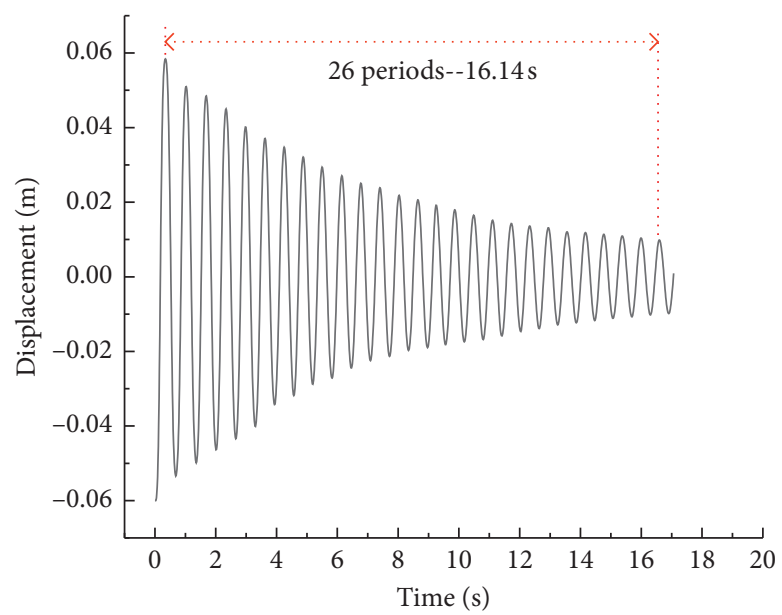

Figure 5: The displacement curve of the rolling ball.

two are in the state of free attenuation vibration. In order to realize the purpose of damping vibration tuned, the input DC current of the damper needs to be $3.1 \mathrm{~A}$. Figure 7 shows the displacement changes of the damper and the high-rise structure when they are in free vibration. According to the displacement change and stiffness $k$ of the structure in free 


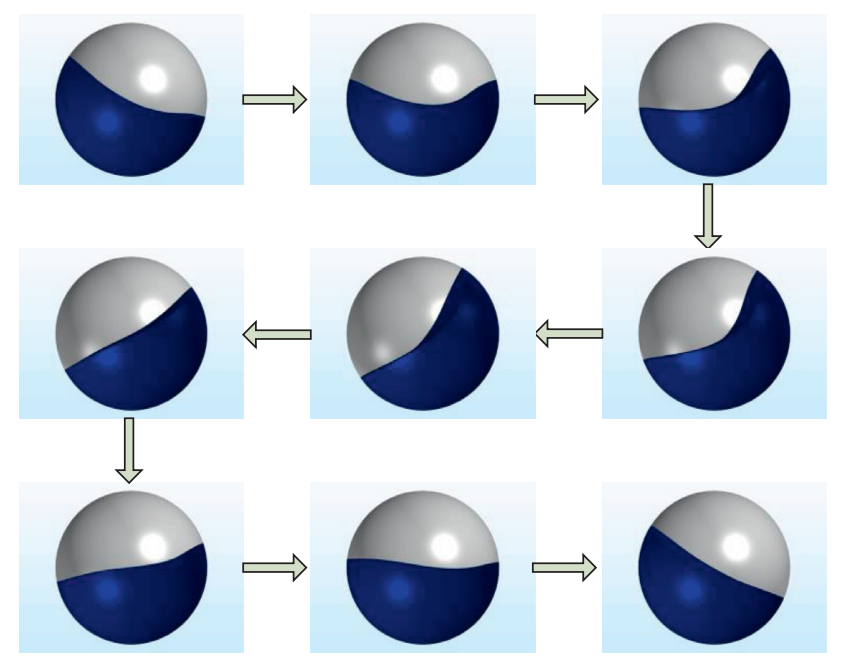

FIgURE 6: The magnetic fluid level change in a vibration period.

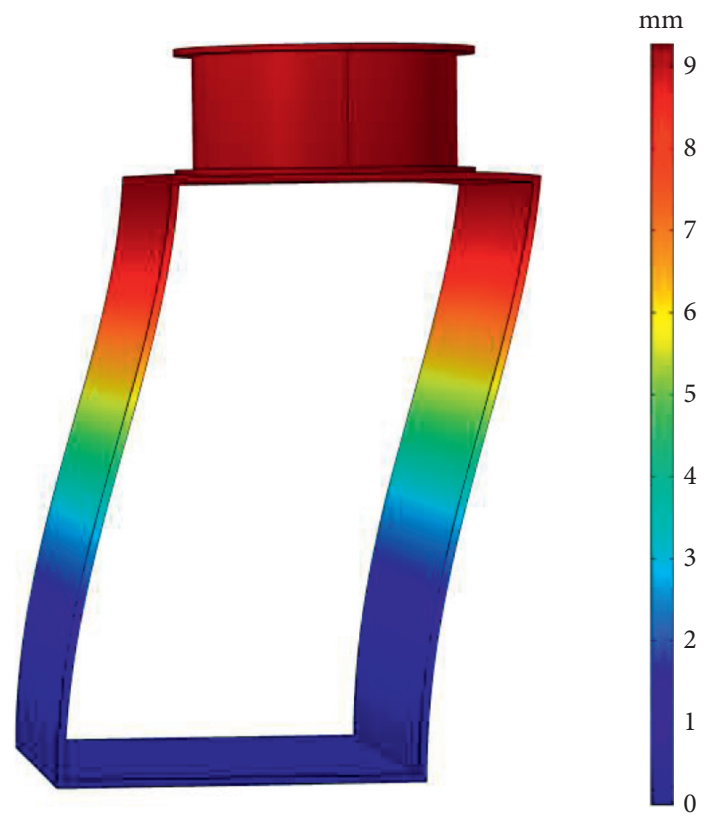

Figure 7: Displacement changes of the damping system in simulation.

vibration attenuation, the elastic recovery force of the structure under free vibration can be obtained by $F=k x$, which is represented by $F_{1}$. At the same time, the horizontal reactive force of damper on the contact surface of high-rise structure is measured, also known as damping force, which is represented by $F_{2}$. Figure 8 shows the force diagram; the two forces are opposite, and Figure 9 shows curves of $F_{1}$ and $F_{2}$ under free vibration. It can be concluded that the maximum damping force of the damper is about $12 \%$ of the vibrating structure elastic force. And with the decrease of the vibration displacement, the structural elastic force decreases, and the damping force provided by the damper also decreases, and finally, the static equilibrium state is achieved. Therefore, the damper can provide appropriate damping force to effectively suppress the vibration of the structure.

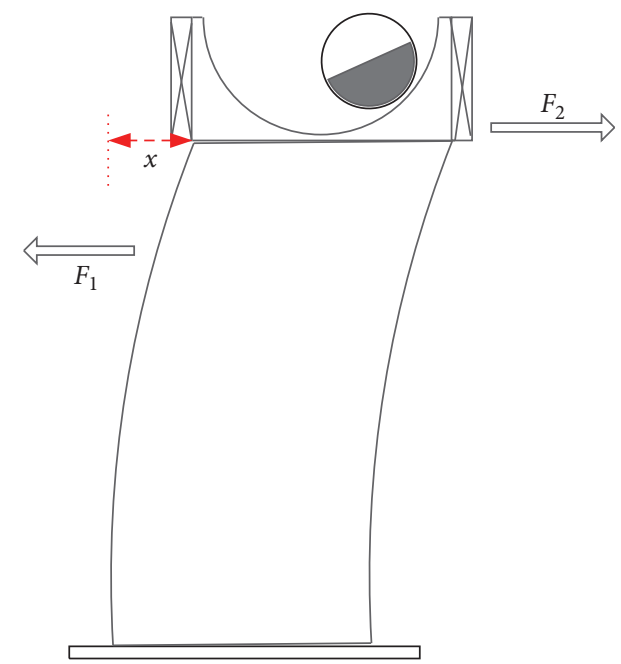

Figure 8: The force diagrams.

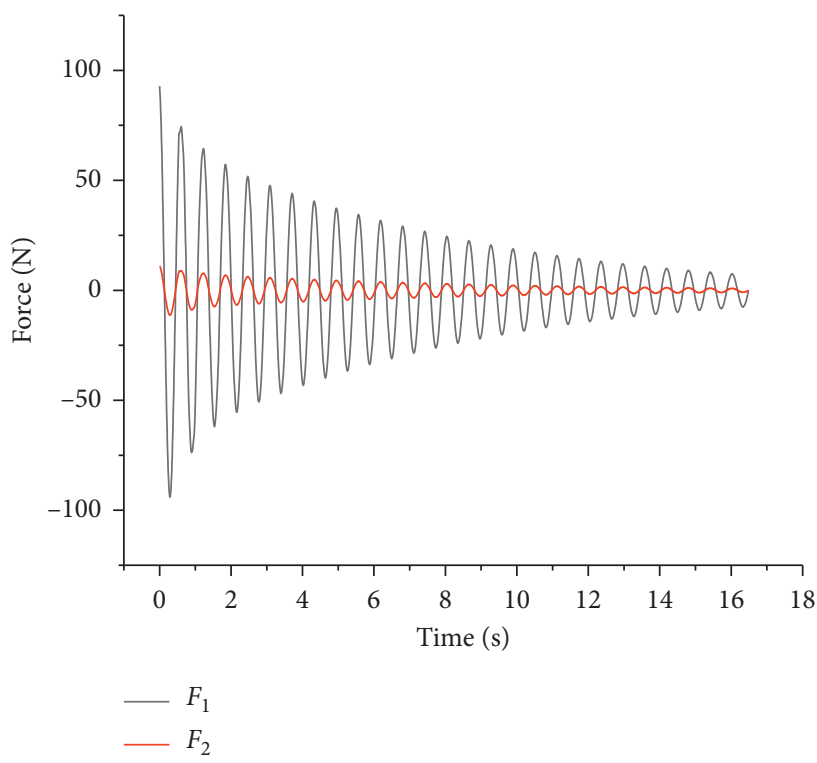

FIgURE 9: The curves of $F_{1}$ and $F_{2}$ under free vibration.

\section{Experiments and Results}

To verify the results of numerical analysis and test the damping performance of the tuned magnetic fluid rollingball damper, a single-layer steel frame structure is used to simulate the single-degree-of-freedom system for the free vibration experiment and harmonic forced vibration experiment. The frame structure is made of $1 \mathrm{~mm}$-thick phosphorous copper plate and the height adjustable range is $300 \mathrm{~mm}$ to $500 \mathrm{~mm}$. During the vibration experiments, the damper and displacement sensor are fixed on the top of the frame, as shown in Figure 10. The experimental model of the tuned magnetic fluid rolling-ball damper is made of resin by $3 \mathrm{D}$ printing, as shown in Figure 11. Its parameters are consistent with the simulation; the radii of groove and ball are $100 \mathrm{~mm}$ and $40 \mathrm{~mm}$, respectively. The diameter of DC coil wire is $1 \mathrm{~mm}$ and the turn number of the coil is 400 . 


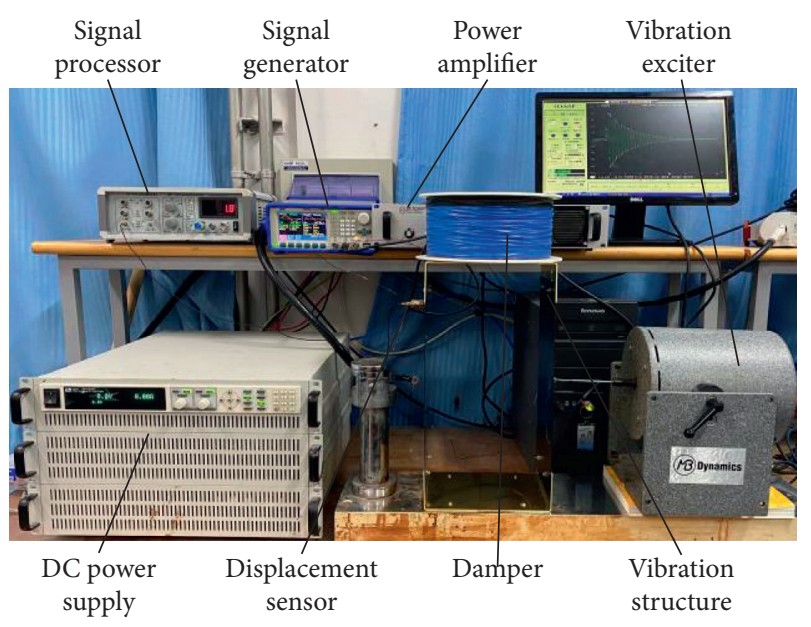

Figure 10: Experimental apparatus.

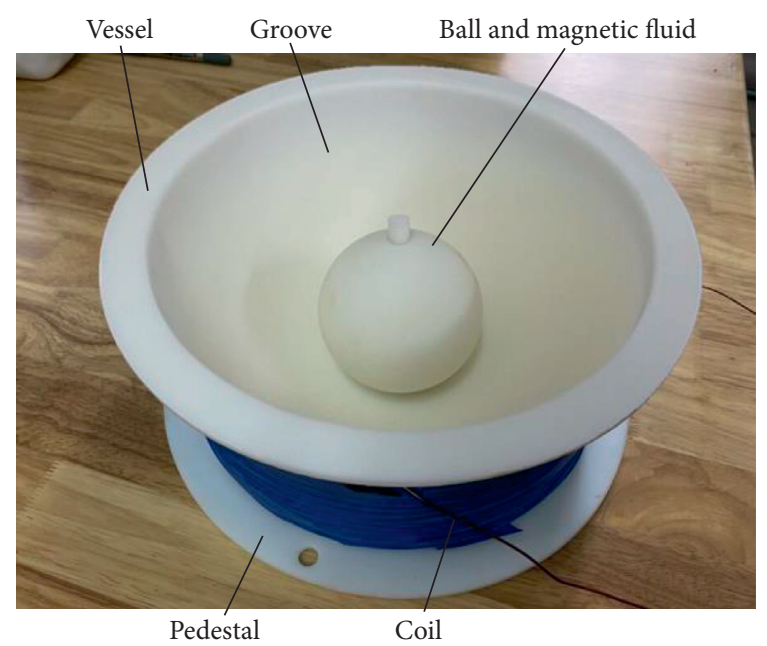

FIgURE 11: The experimental model of the damper.

The two kinds of magnetic fluid used in the experiment are kerosene-based $\mathrm{Fe}_{3} \mathrm{O}_{4}$ magnetic fluid and water-based $\mathrm{Fe}_{3} \mathrm{O}_{4}$ magnetic fluid (supplied by Beijing Shenran Magnetic Fluid Co., Ltd.), both with the volume fraction of $8 \%$, and their density is $1.41 \mathrm{~g} / \mathrm{cm}^{3}$ and $1.46 \mathrm{~g} / \mathrm{cm}^{3}$, respectively. The corresponding dampers are named TMFRBD1 and TMFRBD2, respectively.

The free vibration experiment of the test model adopts the method of the fixed displacement offset, which means that the top of the structure is fixed offset by $12 \mathrm{~mm}$. Free vibration tests are carried out under two conditions: with the damper and without the damper. After the installation of the damper, the first natural frequency of the structure has almost no change. The free vibration frequencies of the structure with different height are measured as $1.61 \mathrm{~Hz}$ and $1.70 \mathrm{~Hz}$. Therefore, to realize the purpose of tuning vibration reduction, the input currents of TMFRBD1 are 3.1 $\mathrm{A}$ and $1.2 \mathrm{~A}$, and the input currents of TMFRBD2 are $3.8 \mathrm{~A}$ and $1.3 \mathrm{~A}$, respectively. Figure 12 shows the amplitude attenuation curve at the top of the vibration structure under

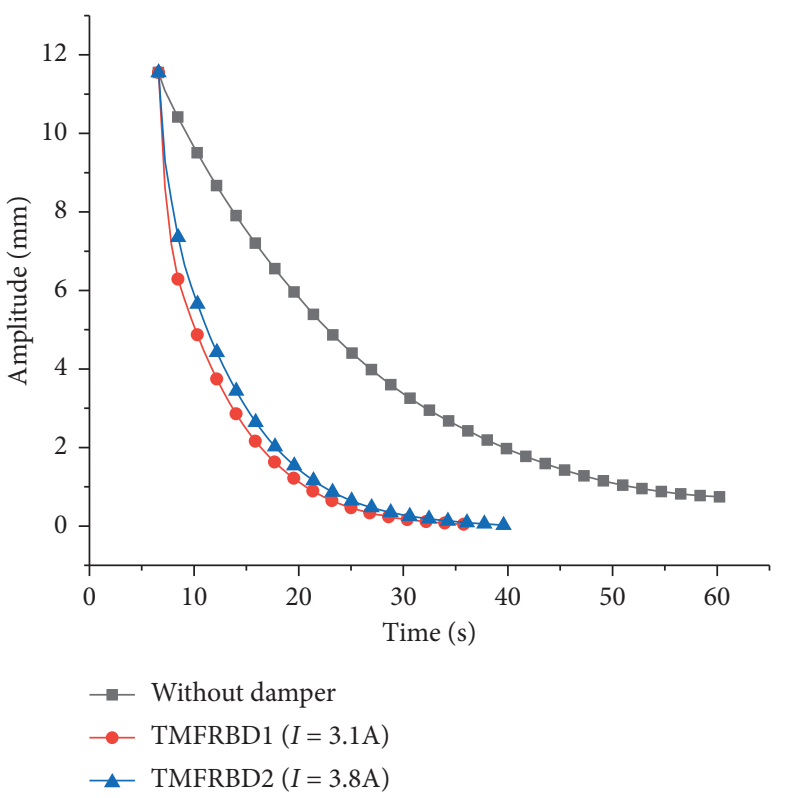

Figure 12: The amplitude attenuation curve under $1.61 \mathrm{~Hz}$.

TMFRBD1 and TMFRBD2 and no damper, when the vibration frequency is $1.61 \mathrm{~Hz}$. TMFRBD1 and TMFRBD2 reduce the free vibration response time of the structure by $40.1 \%$ and $36.6 \%$, respectively, and TMFRBD1 makes the amplitude decay of the structure faster.

The damping ratio of the vibration structure system was obtained by using the logarithmic decay rate method, as shown in Table 1 . When the free attenuation vibration frequency is $1.61 \mathrm{~Hz}$, TMFRBD1 and TMFRBD2 increase the damping ratio of the structural system by $2.23 \%$ and $2.17 \%$, respectively. When the free attenuation frequency is $1.70 \mathrm{~Hz}$, TMFRBD1 and TMFRBD2 increase the damping ratio of the structural system by $1.79 \%$ and $1.75 \%$, respectively. It can be seen that after the damper and the structure are tuned at different frequencies, the damping ratio of the structural system can be increased, the vibration response of the structure can be effectively suppressed, the vibration response time of the structure can be reduced, and the effect of tuning and vibration reduction can be achieved. Moreover, TMFRBD1 has a better vibration suppression effect than TMFRBD2.

The harmonic forced vibration experiment uses the electromagnetic exciter to apply the sine excitation with adjustable frequency and amplitude. When the excitation frequency is $1.66 \mathrm{~Hz}$, to realize the purpose of tuning vibration reduction, the input currents of TMFRBD1 and TMFRBD2 are $2 \mathrm{~A}$ and $2.5 \mathrm{~A}$, respectively. Figure 13 shows the displacement response curve at the top of the vibration structure under TMFRBD1 and TMFRBD2 and no damper, when the excitation frequency is $1.66 \mathrm{~Hz}$. Adjusting the excitation frequency of the shaker and the input current of the damper, the experimental results of tuning vibration reduction at different frequencies are shown in Table 2.

To evaluate the damping effect of the damper on the structural vibration response, a variable is defined as 
TABLE 1: The test results of free attenuation vibration.

\begin{tabular}{llcc}
\hline $\begin{array}{l}\text { Frequency } \\
(\mathrm{Hz})\end{array}$ & Condition & $\begin{array}{c}\text { Current } \\
(\mathrm{A})\end{array}$ & $\begin{array}{c}\text { Damping ratio } \\
(\%)\end{array}$ \\
\hline 1.61 & $\begin{array}{c}\text { Without } \\
\text { damper } \\
\text { With }\end{array}$ & - & 1.24 \\
1.61 & $\begin{array}{c}\text { TMFRBD1 } \\
\text { With } \\
\text { TMFRBD2 } \\
\text { Without } \\
\text { damper } \\
\text { With }\end{array}$ & 3.1 & 3.47 \\
1.70 & $\begin{array}{c}\text { TMFRBD1 } \\
\text { With }\end{array}$ & 1.2 & 3.41 \\
1.70 & TMFRBD2 & 1.3 & 1.18 \\
\hline
\end{tabular}

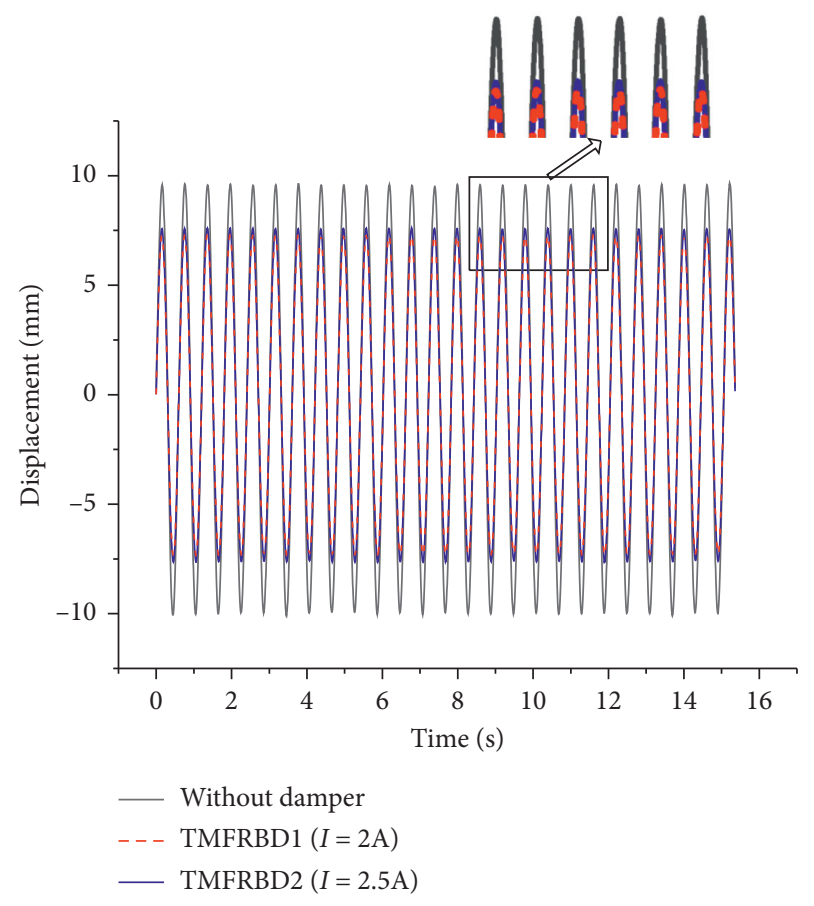

Figure 13: The displacement response curve under $1.66 \mathrm{~Hz}$.

$$
\eta=\frac{\max \left|P_{\text {nodamper }}\right|-\max \left|P_{\text {damper }}\right|}{\max \left|P_{\text {nodamper }}\right|} \times 100 \%,
$$

where $P_{\text {nodamper }}$ is the maximum displacement response of the structure without the control of the damper and $P_{\text {damper }}$ is the maximum displacement response of the structure with the control of the damper.

According to Figure 13 and Table 2, it can be found that the damper can effectively reduce the displacement response of the vibration structure after tuning at different excitation frequencies, and the vibration reduction rate can reach about $20 \%$. Moreover, TMFRBD1 has a better damping performance than TMFRBD2.

Based on the experimental results of free vibration and harmonic forced vibration, comparing TMFRBD1 and TMFRBD2, it can be found that TMFRBD1 has the better
TABLE 2: The test results of harmonic forced vibration.

\begin{tabular}{lcccc}
\hline Condition & $\begin{array}{c}\text { Frequency } \\
(\mathrm{Hz})\end{array}$ & $\begin{array}{c}\text { Current } \\
(\mathrm{A})\end{array}$ & $\begin{array}{c}\text { Amplitude } \\
(\mathrm{mm})\end{array}$ & $\eta(\%)$ \\
\hline Without & - & - & 9.34 & - \\
damper & & 0 & 7.51 & 19.59 \\
TMFRBD1 & 1.76 & 0 & 7.60 & 18.63 \\
TMFRBD2 & 1.76 & 1 & 7.51 & 19.59 \\
TMFRBD1 & 1.71 & 1.1 & 7.59 & 18.73 \\
TMFRBD2 & 1.71 & 2 & 7.61 & 18.52 \\
TMFRBD1 & 1.66 & 2.5 & 7.69 & 17.67 \\
TMFRBD2 & 1.66 & 3 & 7.31 & 21.73 \\
TMFRBD1 & 1.62 & 3.6 & 7.41 & 20.66 \\
TMFRBD2 & 1.62 & 4 & 7.41 & 20.24 \\
TMFRBD1 & 1.57 & 4.9 & 7.56 & 19.06 \\
TMFRBD2 & 1.57 & & &
\end{tabular}

damping performance. The main reason can be concluded as follows: when the solid particles are magnetized, they will overcome the viscous torque and rotate. The higher the viscosity of the base liquid is, the larger the magnetic torque is required and the larger the apparent viscosity of the magnetic fluid is, so the energy dissipation is faster. Kerosene has a higher viscosity relative to water; for the samevolume fraction, the apparent viscosity is higher, and the damping performance is better. Therefore, the kerosenebased $\mathrm{Fe}_{3} \mathrm{O}_{4}$ magnetic fluid is more suitable for the damping liquid of the damper.

\section{Conclusions}

In this study, based on the vibration characteristics of highrise structure, a tuned magnetic fluid rolling-ball damper is presented to suppress the wind-induced vibration of highrise structure. The multiphysical field coupling analysis of the damper is carried out, and the magnetic-fluid-solid multiphysical field coupling model of the damper is established, which can accurately simulate the working characteristics of the damper. Based on the simulation study and vibration damping performance experiments, the following conclusions can be drawn:

(1) The simulation result of the ball's natural rolling frequency is consistent with the theoretical calculation. The magnetic field distribution in the damper meets the requirement of the viscosity change of magnetic fluid and achieves the semiactive control of the damper.

(2) When the structure is in free vibration, after the tuned magnetic fluid rolling-ball damper and the structure are tuned, the maximum damping force of the damper is about $12 \%$ of the elastic force of the structure. And magnetic fluid is in the turbulent state during operation, the friction energy consumption between the magnetic nanoparticles and the carrier liquid is faster, and the vibration reduction effect is better.

(3) According to the experimental results of free vibration and harmonic forced vibration, under the 
volume fraction, the kerosene-based $\mathrm{Fe}_{3} \mathrm{O}_{4}$ magnetic fluid has higher apparent viscosity, faster energy dissipation, and better damping performance as damping liquid. Therefore, the kerosene-based $\mathrm{Fe}_{3} \mathrm{O}_{4}$ magnetic fluid should be used as damping liquid in the tuned magnetic rolling-ball damper for practical projects.

\section{Data Availability}

The data used to support the findings of this study are included within the article.

\section{Conflicts of Interest}

The authors declare that there are no conflicts of interest regarding the publication of this paper.

\section{Acknowledgments}

This work was supported by the National Natural Science Foundation of China under Grant no. 51877066, Natural Science Foundation of Hebei Province under Grant no. E2020208039, and the Hebei Province Innovation Funding Project for Postgraduates in 2020 under Grant no. CXZZSS2020033.

\section{References}

[1] S. Odenbach, "Recent progress in magnetic fluid research," Journal of Physics: Condensed Matter, vol. 16, no. 32, pp. R1135-R1150, 2004.

[2] M. Sheikholeslami, M. M. Rashidi, and D. D. Ganji, "Effect of non-uniform magnetic field on forced convection heat transfer of $\mathrm{Fe}_{3} \mathrm{O}_{4}$-water nanofluid," Computer Methods in Applied Mechanics and Engineering, vol. 294, pp. 299-312, 2015.

[3] W. Yang, Y. Zhai, and X. Yang, "Research on the low-frequency pressure generator based on magnetic fluid," International Journal of Modern Physics B, vol. 33, no. 16, Article ID 1950173, 2019.

[4] G. Thirupathi and R. Singh, "Study of magnetoviscosity of ferromagnetic MnZn-ferrite ferrofluid," IEEE Transactions on Magnetics, vol. 51, no. 11, pp. 1-4, 2015.

[5] M. Izadi, M. Javanahram, S. M. H. Zadeh, and D. Jing, "Hydrodynamic and heat transfer properties of magnetic fluid in porous medium considering nanoparticle shapes and magnetic field-dependent viscosity," Chinese Journal of Chemical Engineering, vol. 28, no. 2, pp. 329-339, 2020.

[6] J. Nowak and S. Odenbach, "Magnetoviscous effect in a biocompatible ferrofluid," IEEE Transactions on Magnetics, vol. 49, no. 1, pp. 208-212, 2013.

[7] M. Victory, R. P. Pant, and S. Phanjoubam, "Synthesis and characterization of oleic acid coated $\mathrm{Fe}-\mathrm{Mn}$ ferrite based ferrofluid," Materials Chemistry and Physics, vol. 240, Article ID 122210, 2020.

[8] G. Paul, P. Kumar Das, and I. Manna, "Synthesis, characterization and studies on magneto-viscous properties of magnetite dispersed water based nanofluids," Journal of Magnetism and Magnetic Materials, vol. 404, pp. 29-39, 2016.

[9] J. Yao, D. Li, X. Chen, C. Huang, and D. Xu, "Damping performance of a novel ferrofluid dynamic vibration absorber," Journal of Fluids and Structures, vol. 90, pp. 190204, 2019.

[10] X.-G. Lin, F. Guo, C.-B. Du, and G.-J. Yu, "The mechanical properties of a novel STMR damper based on magnetorheological silly putty," Advances in Materials Science and Engineering, vol. 2018, pp. 1-15, 2018.

[11] Y.-Q. Guo, J. Zhang, D.-Q. He, and J.-B. Li, "Magnetorheological elastomer precision platform control using offo-pid algorithm," Advances in Materials Science and Engineering, vol. 2020, pp. 1-9, 2020.

[12] S. Chen, J. Huang, H. Shu, T. Sun, and K. Jian, “Analysis and testing of chain characteristics and rheological properties for magnetorheological fluid," Advances in Materials Science and Engineering, vol. 2013, pp. 1-6, Article ID 290691, 2013.

[13] K. Robert and M. Gabor, "Some applications of ferrofluid magnetic colloids," IEEE Transactions on Magnetics, vol. 6, no. 3, pp. 694-698, 1970.

[14] R. Moskowitz, P. Stahl, and W. R. Reed, "Inertia damper using ferrofluid," US Patent US 4123675 A, 1978.

[15] K. Nakatsuka, H. Yokoyama, J. Shimoiizaka, and T. Funaki, "Damper application of magnetic fluid for a vibration isolating table," Journal of Magnetism and Magnetic Materials, vol. 65, no. 2-3, pp. 359-362, 1987.

[16] X. Yang, Q. Yang, W. Yang, B. Guo, and L. Chen, "Analysis of adjustable magnetic fluid damper in DC magnetic field for spacecraft applications," IEEE Transactions on Applied Superconductivity, vol. 28, no. 3, pp. 1-5, 2018.

[17] K.-I. Ohno, M. Shimoda, and T. Sawada, "Optimal design of a tuned liquid damper using a magnetic fluid with one electromagnet," Journal of Physics: Condensed Matter, vol. 20, no. 20, Article ID 204146, 2008.

[18] K.-I. Ohno and T. Sawada, "An effect of vertical sloshing on a fluid pressure and a surface displacement in a tuned magnetic fluid damper," International Journal of Applied Electromagnetics and Mechanics, vol. 33, no. 3-4, pp. 1411-1416, 2010.

[19] K.-I. Ohno, H. Suzuki, and T. Sawada, "Analysis of liquid sloshing of a tuned magnetic fluid damper for single and coaxial cylindrical containers," Journal of Magnetism and Magnetic Materials, vol. 323, no. 10, pp. 1389-1393, 2011.

[20] T. Oyamada, H. Masuda, K. Ikari, T. Sawada, X. Maldague, and T. Takagi, "Damping characteristics of a magnetic fluid tuned liquid column damper under static magnetic fields," International Journal of Applied Electromagnetics and Mechanics, vol. 45, no. 1-4, pp. 659-665, 2014.

[21] J. Yao, J. Chang, D. Li, and X. Yang, "The dynamics analysis of a ferrofluid shock absorber," Journal of Magnetism and Magnetic Materials, vol. 402, pp. 28-33, 2016.

[22] D. Li, J. Yao, and J. Chang, "A novel magnetic fluid shock absorber with levitating magnets," Journal of Vibroengineering, vol. 19, no. 1, pp. 28-37, 2017.

[23] S. Wang, Y. Liu, D. Li, and X. He, "A ferrofluid-based tuned mass damper with magnetic spring," International Journal of Applied Electromagnetics and Mechanics, vol. 60, no. 1, pp. 13-19, 2019.

[24] C. Huang, J. Yao, T. Zhang, Y. Chen, H. Jiang, and D. Li, "Damping applications of ferrofluids: a review," Journal of Magnetics, vol. 22, no. 1, pp. 109-121, 2017.

[25] M. Benedetti, V. Fontanari, and L. Battisti, "Structural health monitoring of wind towers: residual fatigue life estimation," Smart Materials and Structures, vol. 22, no. 4, Article ID 045017, 2013.

[26] A. S. Verma, Z. Jiang, Z. Gao, and N. P. Vedvik, "Effects of a passive tuned mass damper on blade root impacts during the 
offshore mating process," Marine Structures, vol. 72, Article ID $102778,2020$.

[27] H. Zuo, K. Bi, and H. Hao, "Mitigation of tower and out-of-plane blade vibrations of offshore monopile wind turbines by using multiple tuned mass dampers," Structure and Infrastructure Engineering, vol. 15, no. 2, pp. 269-284, 2019.

[28] J. Chen and C. T. Georgakis, "Tuned rolling-ball dampers for vibration control in wind turbines," Journal of Sound and Vibration, vol. 332, no. 21, pp. 5271-5282, 2013.

[29] H. Soltanipour, "Two-phase simulation of magnetic field effect on the ferrofluid forced convection in a pipe considering brownian diffusion, thermophoresis, and magnetophoresis," The European Physical Journal Plus, vol. 135, no. 9, Article ID $702,2020$.

[30] A. Bhandari, "Study of ferrofluid flow in a rotating system through mathematical modeling," Mathematics and Computers in Simulation, vol. 178, pp. 290-306, 2020.

[31] J. Zhao, Z. Ning, and M. Lv, "Large eddy simulation of the two-phase flow pattern and bubble formation process of a flow mixing nozzle under a gas-liquid mode," Fluid Dynamics Research, vol. 51, no. 5, Article ID 055510, 2019. 